\title{
Vaccination Against Atherosclerosis: A Brief Review and Update
}

Hamideh Amirfakhryan*

Faculty of Health Science, University of South Wales, UK

Article Info

\section{Article Notes}

Received: February 11, 2020

Accepted: March 11, 2020

\section{*Correspondence:}

Dr. Hamideh Amirfakhryan, Faculty of Health Science, University of South Wales, UK; 32/4, Los Pacos, Fuengirola, Malaga, Spain; Telephone No: 0034602292911; Email: malihe.amirfakhrian@gmail.com.

(c) 2020 Amirfakhryan $\mathrm{H}$. This article is distributed under the terms of the Creative Commons Attribution 4.0 International License.

\section{Keywords:}

Vaccination

Atherosclerosis

Antigen; Antibody

Clinical trial

Animal

Mice

\section{Abstract}

Vaccination against atherosclerosis as a potential effective approach has been under investigation for more than 20 years. Different antigens have been tested in animals with a great success. Lipid-related antigens like OxLDL, PCSK9, non-lipid related antigens like interleukins, HSPs $\beta 2 \mathrm{GPI}$, DNA vaccination and whole cell vaccination are some examples of successful examinations in animals. Plant-based vaccination which has some advantages over traditional methods has been attracted the scientists' attention recently. Despite the very substantial struggles and promising results during these years, vaccination against atherosclerosis could not be utilized for the treatment of atherosclerosis in human in part due to the lack of clinical trials to access its safety and efficacy. In fact, designing clinical trials with a sufficient number of participants together with a sufficient duration of follow up to explore the influence of vaccine on the prevention and treatment of atherosclerosis seems to be an imperative requisiteness. It looks investing on clinical trials must be a priority to achieve a clear sight regarding the new, tempting, and promising strategy for vaccination against atherosclerosis.

\section{Background}

Despite a lot of efforts to reduce the burden of atherosclerosis, it still remained as the principal reason of death worldwide. Atherosclerosis is an inflammatory disease in which both innate and adaptive immune responses are involving through all the stages of the disease $\mathrm{e}^{1,2}$.

Vaccination against atherosclerosis is a rather new, potential effective approach that has been attracted scientists' attention over 20 years. However, to date, almost all the investigations have been conducted in animals and only very few clinical trials have been

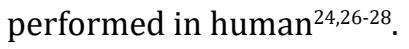

As atherosclerosis is a complex and multifactorial disease, finding proper antigen and adjuvant for vaccination against the disease is not as simple as finding suitable antigens in infectious diseases.

Though, in recent years, utilizing different antigens like oxidized low density lipoprotein (OxLDL), Interleukins (ILs), Proprotein convertase substilisin/kexin type 9 (PCSK9) have been associated with a great success in animal models.

Not like other autoimmune diseases, atherosclerosis begins at the very early ages ${ }^{3}$, one of the characteristics of the disease that persuades investigators to struggle in order to prevent it at the very early phase.

Immunomodulation as a potential strategy to reduce atherosclerosis could be a successful tool if it results in stopping 
and regression of the plaque growth, stabilizing the plaque, and diminishing the inflammatory process associated with atherosclerotic plaque formation ${ }^{3}$. Concerning vaccination against atherosclerosis, there are two approaches which include selective suppression of pro-atherogenic immune response, and selective activation of anti-atherogenic immune response ${ }^{3}$.

In this mini review different antigens and vaccination methods will be briefly discussed. It might be said that now, in this subject finding proper antigen is not a big deal thus far, but designing clinical trials with sufficient human participation, and sufficient follow up to consider the efficacy and safety of successful vaccines in human is the greater apprehension now.

\section{Types of Antigens and Vaccinations}

\section{Lipid-related antigens}

Ox-LDL and apoprotein B100 (Apo B100) derived peptides are at the forefront of antigens used for vaccination, because they have a strong causative link with atherothrombosis. Several studies confirmed the correlation between antiOxLDL antibody titers and the extent of protection against atherosclerosis progression ${ }^{4-7}$. Correspondingly, a positive correlation has been described between the concentration of Ox-LDL and severity of coronary artery disease (CAD) and acute coronary syndrome $(\mathrm{ACS})^{8-10}$.

Antibodies against lipoproteins seem to be both protective and proatherogenic depending on their predominancy. If IgM antibody recognizing epitopes on OxLDL and phosphorylcholine (PC) headgroups on the surface of apoptotic cells would be the predominant antibody, it would be protective against atherosclerosis ${ }^{11-14}$. In contrast, the predominant IgG antibody leads to proatherogenic effects $^{15-18}$.

Among several potential antigenic epitopes within Apo B100, P210 had been the most frequent used antigen in vaccine formulation because of its strong atheroprotective effects $^{20-22}$. The use of immunoreactive peptides like P210 along with P2 and P143 in vaccine formulation have been demonstrated to reduce atherosclerosis by $40-70 \%$ in mice $\operatorname{model}^{19,23}$.

Cholesteryl ester transfer protein(CETP) is a protein involved in reverse cholesterol transfer pathway which explains its atherprotective role. However, its role in transferring cholesteryl ester from high density lipoprotein (HDL) results in reduction of the cholesterol content of HDL, the apoprotein A1 (ApoA1) content, and the size of HDL particle that elucidates the atherogenic role of CETP ${ }^{22,25}$.

Given the controversial role of CETP, the results of the studies in which CETP inhibitors were used for the treatment of atherosclerosis both in animals and human models have shown conflicting results as well. For instance, in the ILLUSTRATE (Investigation of Lipid Level Management Using Coronary Ultrasound to Assess Reduction of Atherosclerosis) trial in which a CETP inhibitor called torcetrapib was prescribed to the patients, no regression of coronary atherosclerosis was observed ${ }^{26}$. However, in post hoc analysis of the study, a significant reduction of coronary atherosclerosis was observed in the highest HDL-cholesterol quartile ${ }^{27}$.

In the ILLUMINATE (Investigation of Lipid Management to Understand its Impact in Atherosclerotic Events) trial torcetrapib plus atorvastatin was compared with atorvastatin plus placebo. The trial was prematurely stopped because of adverse events and deaths in the torcetrapib group representing other novel protective functions of CETP such as protection against lipoprotein oxidation, anti-inflammatory, and anti-adipogenic $\operatorname{activities}^{24,28}$.

PCSK9 is an enzyme which binds to LDL receptor, prohibits it from recycling to the cell surface to uptake more LDL cholesterol, and leads to increase the circulating level of LDL cholesterol. The circulating level of PCSK9 has been demonstrated to correlate with future cardiovascular events $^{30}$. Passive immunization of PCSK9 has been successful through PCSK9 monoclonal antibodies like Alicrumab and evolocumab in reducing LDL cholesterol concentration $^{29}$. However, there are some drawbacks of these drugs such as poor drug adherence due to the need for intermittent injection, high cost, and adverse effects. In contrast, the active immunization against PCSK9 has been investigated and established promising results ${ }^{29,33}$. Landlinger and his colleagues proved the positive effects of the active immunization of mice model with AT04A which mimics N-terminal epitope of mature human and homologue mouse PCSK9 protein. They established a significant decrease in serum level of PCSK9, LDL cholesterol, the necrotic core content, and both area and number of atherosclerotic lesions ${ }^{31,32}$. AT04A vaccine is currently being tested in a phase I clinical trial awaiting for its final results ${ }^{32}$.

However, there are some downsides regarding the peptide antigens such as low immunogenicity that needs to be improved. Adjuvants as useful tools to augment the immunogenicity of the peptide antigens have been utilized by investigators include keyhole limpet hemocyanin $(\mathrm{KLH})^{31}, \mathrm{DNA}^{34}$, and virus-like particles (VLP) ${ }^{35}$ which strongly increased the immunogenicity. However, there are still a lot of concerns regarding their safety and efficiency in human 39 .

In this line, an appropriate strategy to produce strong antibody responses against self-antigens is to present them in a repetitive and highly dense format ${ }^{36,37}$. Liposome 
nanoparticles are safe bilayer spherical vesicles that have been frequently used as adjuvant delivery systems in vaccine formulations. They are feasible carriers for displaying antigens in peptide-based vaccines ${ }^{34}$. It was found that vaccines containing PCSK9 peptide exposed on the nanoliposome surface could induce long-term, strong, safe, and specific antibodies against PCSK9 in BALB/c mice $^{38}$. In this line, Momtazi-Borojeni, et al showed that nanoliposomal PCSK9 vaccine could generate long-lasting, functional, and safe PCSK9-specific antibodies in mice with severe atherosclerosis, which was associated with longterm therapeutic effect against hypercholesterolemia and atherosclerosis ${ }^{39}$.

\section{Non-lipid related antigens}

Apo lipoprotein E (ApoE) is a lipid transport protein which carries out its protective effects through vascular alterations by $\mathrm{N}$-terminus of the protein. However, the function of C-terminus of the protein has been partially defined. Among peptides eluted, Ep1.B (239-252) is a 14-amino acid C-terminal ApoE peptide that is a fragment of a naturally processed peptides (236-252) of murine ApoE. It was demonstrated that Ep1.B injection reduced neointimal hyperplasia after vascular surgery in rats and mice $^{40,60}$.

Heat shock proteins (HSPS) is found on the surface of endothelial cells, mononuclear cells, and vascular smooth muscle cells (VSMCs) in human atherosclerotic plaques ${ }^{41}$. Intranasal vaccination with HSP65 protein has been shown to reduce the development of atherosclerosis ${ }^{42}$.

Interleukins (ILs) have been widely investigated in this regard. Vaccination against IL- $1 \alpha$ which is a powerful proinflammatory cytokine resulted in robust and consistent decrease of atherosclerotic plaque formation in mice model $^{43}$. Furthermore, vaccination against IL-12 (a key inducer of Th1 cells which contributes to the development of atherosclerosis), IL-15 (a pro-inflammatory cytokine expressed in atherosclerotic plaques), and IL-17 (a proinflammatory cytokine with pleiotropic effects on atheroma-associated cell types) have been associated with significant reduction of atherosclerotic plaques burden ${ }^{44-46}$. Conversely, vaccination against P28 subunit of IL-27 has been accompanied with the aggravation of atherosclerosis clarifying the anti-atherogenic properties of P28 subunit of IL-27 via balancing between T helper (Th) 17 cells and T regulatory (Treg) cells ${ }^{46}$.

The retention of LDL in the arterial wall through interaction between ApoB100 protein of LDL and extracellular matrix is a key and early step in the development of atherosclerotic lesion. Fibronectin as an extracellular matrix has been investigated as a potential antigen for vaccination by Duner, et al. They showed that immunization with fibronectin has been associated with significant decrease of atherosclerotic plaque development in mice $^{47}$.

$\beta 2$-glycoprotein I ( $\beta 2$ GPI) which is a highly glycosylated plasma protein has been found to bind apoptotic cells, serving as transporter regulator of cellular traffic and has been implied in atherogenesis ${ }^{48,49}$. In this regard, George, et al examined the human carotid atherosclerotic plaques and observed abundantly expression of $\beta 2 \mathrm{GPI}$ within subendothelial regions and intimal-medial border ${ }^{50}$. Vaccination of mice against $\beta 2$ GPI by Haro, et al showed a significant difference in aortic wall thickness between vaccinated and control groups in favor of vaccinated group $^{48}$.

C5a, a potent protein fragment generated by innate immunity complement component $\mathrm{C} 5$, has proinflammatory properties when it binds with $\mathrm{C} 5 \mathrm{a}$ receptor. Vaccination against $\mathrm{C} 5$ a receptor in mice diminished the atherosclerotic lesion development ${ }^{51,52}$.

\section{DNA vaccination}

Oral DNA vaccination has been considered as an exciting vaccination approach in which an expression plasmid encoding the antigen transfers the genetic material from the carrier to the host phagocytes in the gastrointestinal tract, and then is expressed on the MHC molecule ${ }^{53}$.

Using this method, Hauer and his colleagues used live attenuated bacterium Salmonella. Typhimurium containing vascular endothelial growth factor2 (VEGFR2) encoding plasmid resulting in the significant reduction of atherosclerosis in mice ${ }^{54}$.

Another DNA vaccination approach which has been associated with success in mice was performed by Zhou, et $\mathrm{al}^{55}$. They immunized mice with intramuscular injected vaccine targeted to Fractalkine (CX3CR1) which is an essential chemokine in macrophages recruitment during the pathogenesis of atherosclerosis in both mice and human, and enhanced by dendritic cell targeting using DEC205 single chain variable region fragment. A Significantly reduced atherosclerotic plaque was observed in vaccinated mice, along with less macrophage infiltration, and less lipid deposition in the plaques.

An intra-nasal delivery of DNA vaccine was developed by Yuan, et al in rabbit model of atherosclerosis. For this purpose, they chose chitosan as a gene carrier to prepare the plasmid pCR-X8-HBc-CETP (pCETP) -loaded chitosan nanoparticles, and investigated the potential of chitosan/ pCETP nanoparticles to elicit serum anti-CETP IgG antibodies. They reported a significant detected serum anti-CETP antibody, a significant lower atherogenic index, and significant lesser extent of aortic atherosclerotic lesions in vaccinated rabbits ${ }^{56}$. 


\section{Plant-based vaccination}

Plant-based vaccination as a potential method of vaccination against atherosclerosis, is based on the integration of transgene into the plant cells through direct (introduction of DNA or RNA) or indirect (the employment of plant bacteria and viruses) methods ${ }^{57}$.

Plant-based vaccines in comparison with traditional vaccines have simpler storage, easier rout of administration, more ability to produce more complex proteins with anticipated biological effects, are more cost-effective, and more capable to produce vaccine for a large scale of population ${ }^{57}$.

Few studies showed the efficacy of plant-based vaccination against atherosclerosis in animal models. In this regard, a chimeric protein was obtained in transgenic $N$. tabacum consisting of C-terminal of cholera toxin B (CTB) composed with ApoB100 and CETP epitopes (CTB: p210: CETPe) to aim both the p210 epitope of ApoB100, and amino acids 461-476 at the C-terminal of CETP. The mice were subcutaneously immunized with biomass from the transgenic plants producing CTB: p210: CETPe, and humoral responses against both ApoB100 and CETP epitopes plus human serum proteins were attained ${ }^{59}$.

\section{Whole cell vaccination}

Macrophage foam cells (FCs) play a key role in the initiation and progression of atherosclerosis. Reducing the formation or the removing induction of FCs could ameliorate atherosclerosis. In this regard, Wang and his colleagues have studied the effect of whole cell vaccine using FCs on the development of atherosclerosis in mice model ${ }^{58}$. They observed that subcutaneous injection of the vaccine to mice with atherosclerosis overtly reduced atherosclerotic lesions via raising effective polyclonal antibodies which might selectively bind to the FCs, and induce the antibody-mediated immune response and augment the clearance of FCs in the lesions and subsequently reduce the plaque formation.

\section{Conclusion and Future Perspectives}

Vaccination strategy is an exciting approach to face atherosclerosis and its consequences. However, taking this approach into the routine clinical setting in human includes many challenges. It appears that DNA vaccination in the form of oral or nasal route is a promising strategy from its efficacy and cost point of views. It is expected that in the next couple of years, this form of vaccination would be much more focused. Furthermore, it seems that the first clinical trials will be performed to examine the efficacy and safety of these vaccines in human. Plant-based vaccines are expected to be more lionized in the future to provide cheaper vaccines in a large scale of population. Using a cocktail of antigens to produce powerful multivalent vaccines seems to be a valuable approach.
Finally, vaccination against atherosclerosis is still a myth for a lot of people, and designing clinical trials with a sufficient number of volunteers, a stable financial support, considering an accurate, precise, and preferably non-invasive assessment of atherosclerosis development, determining the age of volunteers to test vaccines, and the period of follow up could convert this myth to reality.

\section{References}

1. Naghavi M, Abajobir AA, Abbafati C, et al. Global, regional, and national age-sex specific mortality for 264 causes of death, 1980-2016: A systematic analysis for the Global Burden of Disease Study 2016. Lancet. 2017; 390: 1151-210.

2. Robertson AKL, Hansson GK. T cells in atherogenesis: For better or for worse? Arterioscler Thromb Vasc Biol .2006; 26: 2421-2432.

3. García-González V, Delgado-Coello B, Pérez-Torres A, et al. Reality of a Vaccine in the Prevention and Treatment of Atherosclerosis. Arch Med Res. 2015; 46(5): 427-37.

4. Palinski W, Miller E, Witztum JL, et al. Immunization of low density lipoprotein (LDL) receptor-deficient rabbits with homologous malondialdehyde-modified LDL reduces atherogenesis (modified lipoproteins/oxidation/autoantibodies/atherosclerosis/immune system). Med Sci. 199592 (3): 821-825.

5. Ameli S, Hultgårdh-Nilsson A, Regnström J, etal. Effect of immunization with homologous LDL and oxidized LDL on early atherosclerosis in hypercholesterolemic rabbits. Arterioscler Thromb Vasc Biol.1996; 16: 1074-1079.

6. Caligiuri G, Nicoletti A, Poirierand B, et al. Protective immunity against atherosclerosis carried by B cells of hypercholesterolemic mice. J Clin Investig. 2002; 109(6): 745-753.

7. Fukumoto M, Shoji T, Emoto M, et al. Antibodies against oxidized LDL and carotid artery intima-media thickness in a healthy population. Arterioscler Thromb Vasc Biol 2000; 20: 703-707.

8. Napoli C, Nigris F, Williams-Ignarro S, et al. Nitric oxide and atherosclerosis: An update Nitric Oxide. Biol Chem. 2006; 15(4): 265279.

9. Ridker PM, Hennekens $\mathrm{CH}$, Roitman-Johnson B, et al. concentration of soluble intercellular adhesion molecule 1 and risks of future myocardial infarction in apparently healthy men. Lancet. 1998; 351(9096): 88-92.

10. Yamaguchi Y, Yoshikawa N, Kagota S, et al. Elevated circulating levels of markers of oxidative-nitrative stress and inflammation in a genetic rat model of metabolic syndrome. Nitric Oxide Biol Chem. 2006;15(4): 380-6.

11. Slot MC, Theunissen R,Van Paassen P, et al. Anti-oxidized low-density lipoprotein antibodies in myeloperoxidase- positive vasculitis patients preferentially recognize hypochlorite-modified low density lipoproteins. Clin Exp Immunol.2007; 149: 257-264.

12. Van Leeuwen M, Damoiseaux J, Duijvestijn A, et al. The therapeutic potential of targeting $B$ cells and anti-oxLDL antibodies in atherosclerosis. Autoimmun Rev. 2009; 9(1): 53-57.

13. Van Leeuwen M, Damoiseaux J, Duijvestijn A, et al.The IgM response to modified LDL in experimental atherosclerosis: Hypochlorite-modified LDL IgM antibodies versus classical natural T15 IgM antibodies. Ann N Y Acad Sci. 2009; 1173(1): 274-279.

14. Rouhl RPW, Van Oostenbrugge RJ, Theunissen ROMFIH, et al. Autoantibodies against oxidized low-density lipoprotein in cerebral small vessel disease Stroke. 2010; 41: 2687-2689.

15. George J, Shoenfeld Y, Afek A, et al. Enhanced fatty streak formation 
in $\mathrm{C} 57 \mathrm{BL} / 6 \mathrm{~J}$ mice by immunization with heat shock protein- 65 . Arterioscler Thromb Vasc Biol. 1999; 19(3): 505-510.

16. George J, Harats D, Gilburd B, et al. Immunolocalization of $\beta 2$ glycoprotein I (apolipoprotein $\mathrm{H}$ ) to human atherosclerotic plaques: Potential implications for lesion progression. Circulation. 1999; 99: 2227-2230.

17. Bergmark C, Wu R, De Faire U, et al. Patients with early-onset peripheral vascular disease have increased levels of autoantibodies against oxidized LDL. Arterioscler Thromb Vasc Biol. 1995; 15: 441445 .

18. Monaco C, Crea F, Niccoli G, et al. Autoantibodies against oxidized low density lipoproteins in patients with stable angina, unstable angina or peripheral vascular disease: Pathophysiological implications. Eur Heart J. 2001; 22(17): 1572-1577.

19. Chyu Y, Zhao X, Reyes OS, et al. Immunization using an Apo B-100 related epitope reduces atherosclerosis and plaque inflammation in hypercholesterolemic apo E (-/-) mice. Biochem Biophys Res Commun. 2005; 338(4): 1982-9.

20. Fredrikson GN, Björkbacka H, Söderberg I, et al.Treatment with apo B peptide vaccines inhibits atherosclerosis in human apo B-100 transgenic mice without inducing an increase in peptide-specific antibodies. J Intern Med. 2008; 264(6): 563-570.

21. Klingenberg $R$, Lebens $M$, Hermansson $A$, et al. Intranasa immunization with an apolipoprotein B-100 fusion protein induces antigen-specific regulatory $\mathrm{T}$ cells and reduces atherosclerosis. Arterioscler Thromb Vasc Biol. 2010; 30: 946-952.

22. Chyu KY, Zhao X, Dimayuga PC, et al. CD8 + T cells mediate the atheroprotective effect of immunization with an ApoB-100 peptide. PLoS One. 2012; 7(2): e30780.

23. Van der Hoek YY, Sangrar W, Côté GP, et al. Binding of recombinant apolipoprotein(a) to extracellular matrix proteins. Arterioscler Thromb A J Vasc Biol. 2011; 14: 1792-1798.

24. Barter PJ, Caulfield M, Eriksson M, et al. Effects of Torcetrapib in Patients at High Risk for Coronary Events. N Engl J Med. 2007; 357: 2109-2122.

25. Barter P. CETP and atherosclerosis. Arterioscler Thromb Vasc Biol. 2000; 20(9): 2029-2031.

26. Nicholls SJ, Tuzcu EM, Brennan DM, et al. Cholesteryl ester transfer protein inhibition, high-density lipoprotein raising, and progression of coronary atherosclerosis: Insights from illustrate (investigation of lipid level management using coronary ultrasound to assess reduction of atherosclerosis. Circulation. 2008; 18(24): 2029-2031.

27. Nissen SE, Tardif JC, Nicholls SJ, et al. Effect of Torcetrapib on the Progression of Coronary Atherosclerosis. N Engl J Med. 2007; 356 1304-1316.

28. Oliveira HCF, De Faria EC. Cholesteryl ester transfer protein: The controversial relation to atherosclerosis and emerging new biological roles. IUBMB Life. 2011; 63(4): 248-57.

29. Chaudhary R, Garg J, Shah N, et al. PCSK9 inhibitors: A new era of lipid lowering therapy. World J Cardiol. 2017; 9(2): 76-91.

30. Vlachopoulos C, Koutagiar I, Terentes-Printzios D, et al. Relationship of PCSK9 levels with indices of vascular function and subclinical atherosclerosis in patients with familial dyslipidemias. Hellenic Cardiol. 2018; 60(2): 124-128.

31. Galabova G, Brunner S, Winsauer G, et al. Peptide-based anti-PCSK9 vaccines-an approach for long-term LDLc management. PLoS One. 2014; E 9(12): e114469.

32. Landlinger C, Pouwer MG, Juno C, et al. The AT04A vaccine against proprotein convertase subtilisin/kexin type 9 reduces total cholesterol, vascular inflammation, and atherosclerosis in APOE*3Leiden.CETP mice. Eur Heart J. 2017; 38 (32): 2499-2507.
33. Fattori E, Cappelletti M, Surdo PL, et al. Immunization against proprotein convertase subtilisin-like/kexin type 9 (PCSK9) lowers plasma LDL-cholesterol levels in mice. J Lipid Res. 2012; jlr: M028340.

34. Zamani P, Momtazi-Borojeni AA, Nik ME, et al. Nanoliposomes as the adjuvant delivery systems in cancer immunotherapy. J Cell Physiol. 2018; 233(7): 5189-99.

35. Pan Y, Zhou Y, Wu H, et al. A therapeutic peptide vaccine against PCSK9. Sci Rep. 2017; 7(1): 12534

36. Bachmann MF, Dyer MR. Therapeutic vaccination for chronic diseases: a new class of drugs in sight. Nat Rev Drug Discov. 2004; 3(1): 81.

37. Nakagami $H$, Koriyama $H$, Morishita R. Therapeutic vaccines for hypertension and dyslipidemia. Int Heart J. 2014; 55(2): 96-100.

38. Momtazi-Borojeni AA,Jaafari MR, Badiee A, et al. Long-term generation of antiPCSK9 antibody using a nanoliposome-based vaccine delivery system. Atherosclerosis. 2019; 283: 69-78.

39. Momtazi-Borojeni AA, Jaafari MR, Badiee A. et al. Therapeutic effect of nanoliposomal PCSK9 vaccine in a mouse model of atherosclerosis. BMC Med. 2019; 17: 223.

40. Bocksch L, Rider BJ, Stephens T, et al. C-terminal apolipoprotein E-derived peptide, Ep1.B, displays anti-atherogenic activity. Atherosclerosis. 2007; 194(1): 116-24.

41. Kleindienst $\mathrm{R}, \mathrm{Xu} \mathrm{Q}$, Willeit J, et al. Immunology of atherosclerosis. Demonstration of heat shock protein 60 expression and $T$ lymphocytes bearing alpha/beta or gamma/delta receptor in human atherosclerotic lesions. Am J Pathol.1993; 142(6): 1927-37.

42. Long J, Lin J, Yang X, et al. Nasal immunization with different forms of heat shock protein-65 reduced high-cholesterol-diet-driven rabbit atherosclerosis. Int Immunopharmacol. 2012; 13(1): 82-87.

43. Tissot AC, Spohn G, Jennings GT, et al. A VLP-based vaccine against interleukin- $1 \alpha$ protects mice from atherosclerosis. Eur J Immunol. 2013; 43(3): 716-722.

44. Van Es T, Van Puijvelde GHM, Michon IN, et al. IL-15 aggravates atherosclerotic lesion development in LDL receptor deficient mice Vaccine. 2011; 29(5): 976-983.

45. Hauer AD, Uyttenhove C, De Vos P, et al. Blockade of interleukin-12 function by protein vaccination attenuates atherosclerosis. Circulation.2005; 112(7): 1054-1062.

46. Thomas Van ES. Vaccination against atherosclerosis. Division Biopharmaceutics Center for Drug Research (LACDR): Faculty of Science, Leiden University, Leiden/Amsterdam. 2009

47. Dunér P, To F, Beckmann K, et al. Immunization of apoE -/- mice with aldehyde-modified fibronectin inhibits the development of atherosclerosis. Cardiovasc Res. 2011; 91(3): 528-536.

48. De Haro J, Esparza L, Bleda S, et al. Attenuation of early atherosclerotic lesions by immunotolerance with $\beta 2$ glycoprotein $I$ and the immunomodulatory effectors interleukin 2 and 10 in a murine model. J Vasc Surg. 2015; 62(6): 1625-1631.

49. Hasunuma Y, Matsuura E, Makita Z, et al. Involvement of beta2glycoprotein I and anticardiolipin antibodies in oxidatively modified low-density lipoprotein uptake by macrophages. Clin Exp Immunol. 2003; 107(3): 569-573.

50. Jacob G, Dror H, Gilburd B, et al. Immunolocalization of $\hat{I}^{2} 2$ Glycoprotein I (Apolipoprotein H) to Human Atherosclerotic Plaques Potential Implications for Lesion Progression. Circulation. 1999; 99: 2227-30.

51. Ippel JH, De Haas CJC, Bunschoten A, et al. Structure of the tyrosinesulfated $\mathrm{C} 5 \mathrm{a}$ receptor $\mathrm{N}$ terminus in complex with chemotaxis inhibitory protein of Staphylococcus aureus. J Biol Chem. 2009; 284(18): 12363-12372. 
52. Lu X, Xia M, Endresz V, et al. Immunization with a combination of 2 peptides derived from the c5a receptor significantly reduces early atherosclerotic lesion in ldlrtm1her Apobtm2Sgy j mice. Arterioscler Thromb Vasc Biol. 2012; 32: 2358-2371.

53. Batten M, Li J, Yi S, et al. Interleukin 27 limits autoimmune encephalomyelitis by suppressing the development of interleukin 17-producing T cells. Nat Immunol. 2006; 7: 929-936.

54. Hauer AD, Van Puijvelde GHM, Peterse N, et al.Vaccination against VEGFR2 attenuates initiation and progression of atherosclerosis. Arterioscler Thromb Vasc Biol. 2007; 27: 2050-2057.

55. Zhou JJ, Wang YM, Lee VWS, et al. DEC205-DC targeted DNA vaccine against CX3CR1 protects against atherosclerosis in mice. Plos One. 2018; 13(4): e0195657.

56. Yuan X, Yang X, Cai D, et al. Intranasal immunization with chitosan/
pCETP nanoparticles inhibits atherosclerosis in a rabbit model of atherosclerosis. Vaccine. 2018; 26(29-30): 3727-3734.

57. Laere E, Ling APK, Wong YP, et al. Plant-based vaccines: Production and challenges. J Bot. 2016; 2016: 1-11.

58. Wang F, Zhang Z, Fang A, et al. Macrophage Foam Cell-Targeting Immunization Attenuates Atherosclerosis. Front Immunol. 2019; 9: 3127.

59. Salazar-Gonzalez JA, Rosales-Mendoza S, Romero-Maldonado A, et al. Production of a plant-derived immunogenic protein targeting ApoB100 and CETP: toward a plant-based atherosclerosis vaccine. Mol Biotechnol. 2014; 56: 1133-1142.

60. Jun L, Jie L, Dongping Y, et al. Effects of nasal immunization of multitarget preventive vaccines on atherosclerosis. Vaccine. 2012; 30(6): 1029-1037. 